\title{
Erratum
}

\section{Tribological Properties of Motor Oil with Nanodiamond Additive tested for steel friction surfaces with different hardnesses - ERRATUM}

M. Ivanov, S. Smirnov, S. Pavlyshko, D. Ivanov, D. Brenner, and O. Shenderova

doi: 10.1557/opl.2013.1146, Published by Materials Research Society, 26 November 2013.

The article by Ivanov et al. was published with the incorrect volume number. The correct volume number is Volume 1597. The Materials Research Society and Cambridge University Press apologize to the authors for this error. The correct version of the article follows this notice.

\section{Reference}

M. Ivanov, S. Smirnov, S. Pavlyshko, D. Ivanov, D. Brenner and O. Shenderova (2013). Tribological Properties of Motor Oil with Nanodiamond Additive tested for steel friction surfaces with different hardnesses. MRS Online Proceedings Library, 1597, jsapmrs13-15976038 doi:10.1557/opl.2013.1146. 